\title{
"UBI PROCEDITUR EX PRAESUMPTIONIBUS MINUITUR POENA". QUALITÀ DELLA PROVA E QUANTITÀ DELLA PENA SECONDO LA DOCTRINA INNOCENTII
}

\author{
"UBI PROCEDITUR EX PRAESUMPTIONIBUS MINUITUR POENA". \\ QUALITY OF THE EVIDENCE AND QUANTITY OF THE PENALTY \\ ACCORDING TO THE DOCTRINA INNOCENTII
}

\author{
Marta Rossi Doria \\ Università degli Studi di Milano-Bicocca
}

\begin{abstract}
English: In Roman-Canonical inquisitorial process, a strict application of the law of proof would have made it impossible to convict a defendant on the basis of circumstantial evidence. This posed many obstacles to the administration of a criminal justice system oriented to the principle of public interest in the prosecution of crimes. A statement of Innocent IV found in the commentary to c. Quia verisimile (X 2.23.10) opened the way to presumptive conviction: the judge could condemn the defendant in the absence of full evidence, as long as he reduced the sentence. In its original formulation, Innocent's sentence represented no more than a cautionary recommendation. It was the doctrinal elaboration that transformed it, beyond the intentions of its author, into an instrument capable of affecting the theoretical rigidity of the system, admitting discretion in modulating the penalty and applying a punishment even in situations of lack of evidence. In this debate, which the present study intends to analyze in its fundamental stages, the contribution of the decretalists has had a leading weight: this way, too, the central role assumed by canonical teaching in the doctrinal reflection on the theory of the law of proof is revealed.
\end{abstract}

Keywords: Romano-canonical procedure; criminal justice; presumptions; lack of evidence; probatio presuntiva; extraordinary punishment; Innocent IV

Abstract Italiano: Nel processo inquisitorio romano-canonico, una rigida applicazione delle regole del sistema di prova legale non avrebbe reso possibile la condanna di un imputato gravato da soli indizi e presunzioni, ponendo non pochi ostacoli all'amministrazione della giustizia criminale, orientata al principio dell'interesse pubblico alla persecuzione dei reati. Un'affermazione di Innocenzo IV contenuta nel commento al c. Quia verisimile (X 2.23.10) aprì la via alla condanna presuntiva: il giudice avrebbe potuto condannare l'imputato in assenza di piena prova, a patto di infliggere una pena ridotta. Nella sua formulazione originale la massima innocenziana non rappresentava più che una raccomandazione di prudenza. Fu l'elaborazione dottrinale a trasformarla, oltre le intenzioni del suo autore, in uno strumento capace di incidere sulla teorica rigidità del sistema di prova legale, ammettendo discrezionalità nel modulare la pena e nell'applicarla anche in

* Italian Review of Legal History, 7 (2021), n. 1, pagg. 1-29

* https://riviste.unimi.it/index.php/irlh/index

* ISSN 2464-8914 - DOI 10.54103/2464-8914/16880. Articolo pubblicato sotto Licenza CC-BY. 
situazioni di carenza di prova. In questo dibattito, che il presente studio intende analizzare nelle sue tappe fondamentali, il contributo dei decretalisti ha avuto un peso di primo piano: anche per tale via si manifesta il ruolo centrale assunto dalla scienza canonistica nella riflessione dottrinale sulla teoria della prova legale.

Parole chiave: processo romano-canonico; giustizia criminale; presunzioni; difetto di prova; probatio presuntiva; pena straordinaria; Innocenzo IV

Sommario: 1 . Premessa. - 2. Sistema di prova legale e valore probatorio delle presunzioni. - 3. Difetto di prova e inflizione della pena: la doctrina Innocentii. - 4. Oscillazioni della dottrina intermedia: verso una communis opinio. -5 . Oltre la scienza canonistica: la fortuna della doctrina Innocentii tra giurisprudenza consiliare e pratiche criminali.

\section{Premessa}

Il processo medievale regolato dal sistema della prova legale si imperniava, come è noto, su una serie di formalismi predeterminati, che non poche difficoltà opponeva ad una celere amministrazione della giustizia ${ }^{1}$.

I giudici si trovavano apparentemente prigionieri di un sistema probatorio dominato da un importante principio garantista: per giungere a sentenza erano necessarie prove luce clariores ${ }^{2}$. In effetti, però, soprattutto nell'ambito criminale (posto l'interesse pubblico che vi era connesso ${ }^{3}$ ), dottrina e prassi si adoperarono per facilitare il più possibile il funzionamento della macchina processuale, pur nel rispetto formale delle regole romano-canoniche. Furono individuati strumenti utili a consentire una - supposta - più efficace repressione del crimine: in primo luogo la tortura ${ }^{4}$, ma anche, ad esempio, la pena straordinaria, che consentiva al giudice di modulare la sanzione sulla base di poteri arbitrari ${ }^{5}$.

\footnotetext{
${ }^{1}$ Come ha opportunamente notato Rosoni, 1995, p. 124, da solo e di per sé il sistema non avrebbe permesso il funzionamento della macchina processuale.

${ }^{2}$ Lo prescriveva la I. Sciant cuncti (C. 4.19.25, canonizzata in C. 2 q. 8 c. 2), su cui ci soffermeremo a breve (v. infra, nt. 22).

${ }^{3}$ In conformità al principio rei publicae interest ne maleficia remaneant impunita-espresso da Innocenzo III in due decretali, la Inauditum del 1199 e la Ut famae del 1203, poi confluita nel Liber Extra (X 5.39.35) -, su cui v. Sbriccoli, 1998, Landau, 2012, e Varalda, 2016. ${ }^{4}$ Dell'uso della tortura nell'età del diritto comune si è occupato innanzi tutto Fiorelli, 1954; sul tema anche Sbriccoli, 1991 (=2009); Schmoeckel, 2000, pp. 235-267; Durand, Otis-Cour (eds.), 2002, con attinenza al nostro tema in particolare gli studi di Schmoeckel, pp. 315-329 e di Meccarelli, pp. 677-707; Garlati, 2011; Fiori, 2013, pp. 461-488; Chiodi, 2013, 2014 e 2016.

${ }^{5}$ Carbasse, 2014, p. 255, ha evidenziato che se da un lato il sistema vincolava sul piano della ricerca della prova, ampi poteri arbitrari agevolavano il giudice su quello dell'inflizione della pena. La poena extraordinaria, ben lungi dal trovare applicazione nei soli casi in cui mancasse una previsione espressa riguardo al modus puniendi, assunse il carattere
} 
Il problema della modulazione delle pene non poteva però prescindere del tutto da quello della modulazione delle prove. Anche la valutazione della prova raggiunta per via inferenziale - ossia la prova presuntiva - giocò infatti un ruolo importante come elemento di flessibilità del sistema, pur senza sovvertire il paradigma della prova legale ${ }^{6}$. Si ammise la possibilità di condannare in assenza di reali riscontri probatori, a condizione però che la sanzione fosse conseguentemente ridotta.

La letteratura giuridica tedesca indicherà con il concetto di Verdachtstrafe, ovvero "pena per il sospetto" fine del Cinquecento ${ }^{8}$, ma che - in effetti - affonda le sue radici in epoca medievale, ed in ambito canonistico. Fu la massima ubi ex praesumptionibus proceditur multum debet iudex temperare sententiam, formulata da Innocenzo IV, ad aprire la via alla possibilità di condanna in assenza di piena prova. Tale principio, noto ai giuristi come doctrina Innocentii, produsse i suoi effetti ben oltre il diritto canonico classico e divenne, nel tempo, un punto fermo per ogni dissertazione in tema di prova e presunzioni.

L'effetto esercitato sulle dinamiche interne al processo inquisitorio da tale dottrina è stato recentemente paragonato da Mathias Schmoeckel a quello dirompente del crollo di una diga ${ }^{9}$. Nonostante questa riconosciuta importanza, la doctrina Innocentii è stata però relativamente poco studiata dalla storiografia giuridica medievistica ${ }^{10}$. Senza la pretesa di seguirne i secolari sviluppi storici, il

fondamentale di valvola di aggiornamento e correzione dell'intero sistema repressivo, volta alla riforma dell'ordinamento esistente, cfr. ${ }^{B}$. Schnapper, 1973 . Sul tema hanno fatto luce diversi studi utili a comprendere il ruolo cardine che l'arbitrium giudiziale assunse in un sistema processuale altrimenti di difficile, se non impossibile, celebrazione: Cortese, 1978 e 2008; Durand, 1993; Lesne-Ferret, 1996; Meccarelli, 1998; Schmoeckel, 2000, pp. 267-294; Vallerani, 2010, pp. 117-148. Da ultimo Cerrito, 2020.

${ }^{6}$ Alessi, 1979; Schmoeckel, 2000, cfr. in particolare pp. 295-300 e 322 ss.

${ }^{7}$ Sull'impiego della Verdachtsstrafe in epoca moderna si vedano Schaffstein, 1989; Thäle, 1993, e Elemér, 2009. Langbein, 1977, pp. 47-48, ha messo in guardia sull'ingannevolezza del termine, che non trova corrispondenti nel linguaggio giuridico latino: "Writers called it the Verdachtsstrafe, the punishment for suspicion. ... In truth, the Verdachtsstrafe was not a punishment for mere Verdacht, for being suspicious. It was a punishment imposed by the court when the court was persuaded that the accused was guilty, but when his guilt could not be established under the Roman-canon law of proof". L'autore ha anche rinvenuto nell'istituto, che permetteva di punire il crimine senza ricorrere alla confessione, i semi dell'abolizione della tortura. Contro tale interpretazione Schmoeckel, 2000, p. 334.

${ }^{8}$ Langbein, 1977, p. 48; Roth, 1998.

${ }^{9}$ Schmoeckel, 2000, p. 334: «Angesichts des beschriebenen Drucks auf den Richter, im Inquisitionsprozeß zur Verurteilung des vermeintlich Schuldigen zu gelangen, mußte sich Innozenz' Ansatz der Verdachtsstrafe wie bei einem Dammbruch auswirken».

${ }^{10}$ Pur con significative eccezioni: Engelmann, 1922, p. 94; Alessi, 1979, 52 ss.; Di Renzo Villata, 1996, pp. 461-463; Schmoeckel, 2000, pp. 331 ss.; Fiori, 2016, pp. 217 ss. 
presente studio si propone l'obiettivo di ricostruire le principali tappe della sua elaborazione da parte della canonistica dell'età di mezzo: perché la doctrina Innocentii sviluppatasi nella tradizione giuridica, nonostante la denominazione, non corrisponde completamente all'originario pensiero innocenziano. Attraverso di essa la scienza giuridica seppe contribuire, per gradi successivi, alla lenta e progressiva creazione di uno spazio intermedio tra colpevolezza e innocenza, prova e mancanza di essa, condanna e assoluzione.

\section{Sistema di prova legale e valore probatorio delle presunzioni}

Come è noto, il secolo XIII rappresentò uno spartiacque nella storia del processo e del diritto criminale. II pontificato di Innocenzo III (1198-1216) fu segnato da due innovazioni che trasformarono profondamente il sistema processuale in vigore: l'abbandono del giudizio ordalico ${ }^{11}$ e l'introduzione del modello inquisitorio ${ }^{12}$. Si tratta di fenomeni ampiamente studiati dalla storiografia, strettamente collegati tra loro ed entrambi fondanti i successivi sviluppi della procedura criminale. II superamento della procedura ordalica aveva accompagnato sin dall'epoca gregoriana il ritorno ai princìpi del processo romano tramandati dalla compilazione giustinianea. Tuttavia, alla nascita del processo romano-canonico non si accompagnò la riaffermazione di quello che - con espressione moderna - chiamiamo il libero convincimento del giudice, che pure aveva ispirato il regime probatorio romano ${ }^{13}$.

Un noto rescritto di Adriano lasciava al giudice la possibilità di valutare cosa egli credesse vero e cosa, al contrario, sembrasse essere non sufficientemente provato $^{14}$, ma l'imponente mole del Corpus luris Civilis non mancava di fornire anche spunti di formalismo probatorio su cui il medioevo avrebbe edificato la propria dottrina. Giustiniano, ad esempio, aveva ritenuto necessario porre alcune limitazioni alla facoltà di testimoniare, nella convinzione che la scaltrezza degli uomini rendesse tale mezzo spesso utile ad occultare la verità, anziché a farla venire alla luce ${ }^{15}$; esse andavano ad aggiungersi ai divieti già in essere, che

${ }^{11}$ Cfr. in proposito Bartlett, 1986; Fraher, 1992; Baldwin, 1994; McAuley, 2006.

12 Sulle radici canonistiche dell'ordo inquisitorio, dopo Trusen, 1988, pp. 168-230, cfr. Fraher, 1992; Kéry, 2001, pp. 226-268; Fiori, 2012, pp. 351-367; Fiori, 2013, pp. 373-395; Chiodi, 2018, pp. 281-305. Sul processo inquisitorio canonico anche Dezza, 1989; Dezza, 2013, pp. 1-18 e passim.

${ }^{13}$ Provera, 1979, pp. 391-399; Russo, 2017, p. 17. Sul processo civile: Kaser, Hackl, 1996, pp. 361 ss. Sul versante penale: Carbasse, 2000, p. 73.

${ }^{14}$ D. 22.5.3.2, de testibus, I. Testium fides § Eiusdem: «Quae argumenta ad quem modum probandae cuique rei sufficiant, nullo certo modo satis definiri potest. ... Hoc ergo solum tibi rescribere possum summatim non utique ad unam probationis speciem cognitionem statim alligari debere, sed ex sententia animi tui te aestimare oportere, quid aut credas aut parum probatum tibi opinaris».

${ }^{15}$ Nov. 90 , de testibus. 
avevano escluso la testimonianza di chi non potesse vantare una fama integra, degli schiavi, dei nemici dell'accusato ${ }^{16}$. Quella che diverrà la celebre massima testis unus testis nullus era poi fondata in principio su una costituzione di Costantino $^{17}$, a sua volta derivata da precetti biblici ${ }^{18}$, che imponeva di non ammettere la testimonianza di un singolo testimone, indipendentemente dalla sua dignità ed affidabilità (etiam si praeclarae curiae honore praefulgeat). Altre indicazioni riguardavano l'inflizione della tortura, da non disporre senza una base di prova ${ }^{19}$, il valore degli atti scritti ${ }^{20}$, pari a quello della testimonianza, e del giuramento ${ }^{21}$. Gli imperatori Giustiniano, Valentiniano e Teodosio avevano infine formulato una massima generale che invitava gli accusatores a fornirsi di testi idonei, documenti o indizi indubitati e più chiari della luce stessa:

Sciant cuncti accusatores eam se rem deferre debere in publicam notionem, quae munita sit testibus idoneis vel instructa apertissimis documentis vel indiciis ad probationem indubitatis et luce clarioribus expedita. ${ }^{22}$

Essa venne regolarmente citata dagli interpreti al fine di escludere il valore probatorio di ogni circostanza su cui avrebbe astrattamente potuto allungarsi l'ombra del dubbio.

Proprio il dubbio sembrava essere il nemico principale del sistema romano-canonico di prova legale ${ }^{23}$, che si caratterizzava per la determinazione di una gerarchia di prove estremamente dettagliata e, in apparenza, meccanicamente applicabile. Nell'elaborazione di tale teoria, dottrina e legislazione canonica giocarono un ruolo importantissimo, ampiamente evidenziato dalla storiografia ${ }^{24}$ : su una materia già esaustivamente trattata non vale qui la pena soffermarsi, ma è opportuno

${ }^{16}$ Questi ed altri casi si trovano in D. 22.5, de testibus.

${ }^{17}$ C. 4.20.9, de testibus, I. Iurisiurandi. Sulla deposizione dell'unus testis cfr. Padoa Schioppa, 1967 (=2003); Gouron, 1995b (=2000, IX); Treggiari, 2007.

${ }^{18}$ Deut. 17:6 e 19:15

${ }^{19}$ D. 48.18.1.1, de quaestionibus, I. In criminibus eruendis $\S$ Verba rescripti; D. 48.18.18.2, de quaestionibus, I. Unius facinoris, § In ea causa.

${ }^{20}$ C. 4.21.15, de fide instumentorum, I. In exercendis litibus.

${ }^{21}$ D. 12.2.11.3, de iureiurando, I. Sed si possessori § Si cum de hereditate.

${ }^{22}$ C. 4.19.25, de probationibus, I. Sciant cuncti (canonizzata in C. 2 q. 8 c. 2). II concetto, già chiaro, fu enfatizzato dalla glossa: con l'aggiunta dell'attributo meridiana si finì per esigere, a supporto dell'accusa, indizi indubitati e più chiari della luce del sole di mezzogiorno. ${ }^{23}$ La letteratura sul tema è ampia: tra i contributi più risalenti mi limito a ricordare Salvioli, 1927, pp. 465 ss.; Lévy, 1939 e 1965. Tra i più recenti si segnalano gli studi che si sono concentrati su vari aspetti problematici della testimonianza: Mausen, 2006; Chiodi 2013 e 2017; Bassani 2012 e 2017.

${ }^{24}$ Per i più recenti studi sull'influsso che il diritto canonico esercitò sul processo medievale si rimanda ai volumi curati da Condorelli, Roumy, Schmoeckel, Mausen, 2009, 2012 e 2014. 
dedicare una parentesi al più discusso dei mezzi di prova, le praesumptiones ${ }^{25}$.

Parallelamente ad una classificazione delle presunzioni di natura civilistica, che risulterà familiare al lettore moderno e che distingueva tra praesumptiones hominis e legis (la quale, a sua volta, poteva dirsi iuris tantum o iuris et de iure a seconda che ammettesse o meno prova contraria), si andò definendo in ambito canonistico una tripartizione di differente natura ${ }^{26}$. Essa graduava le presunzioni sulla base della loro credibilità: poteva dirsi temeraria una presunzione fondata su connessioni inverosimili ed azzardate, e pertanto inaccettabili in giudizio; la praesumptio probabilis, sorta in relazione alla sua idoneità ad imporre che il sospettato si purgasse dall'accusa prestando giuramento di innocenza ${ }^{27}$, venne in un secondo momento parificata ad una probatio semiplena ${ }^{28}$; la praesumptio violenta, infine, aveva l'effetto di condurre a sentenza. Le definizioni furono comunque varie ed intercambiabili: fere infinitae sunt species praesumptionis, dirà il Panormitano ${ }^{29}$.

È facile intendere come all'interno della gerarchia delle prove la presunzione abbia rappresentato un concetto trasversale, sul cui valore in giudizio, come si vedrà, i dottori si sarebbero interrogati a lungo.

\section{Difetto di prova e inflizione della pena: la doctrina Innocentii}

Nel Liber Extra, al titolo de praesumptionibus, diversi canoni indicavano chiaramente la possibilità di giungere a condanna sulla base di indizi e congetture. Primo e più eloquente era il c. Afferte ${ }^{30}$, che riproponeva la famosa sentenza di Salomone, chiamato a definire la disputa tra due donne che si contendevano un solo figlio. II re, non supportato da alcuna prova determinante, si era avvalso della sua proverbiale capacità di giudizio per giungere a sentenza sulla base della sola presunzione che tra le due la vera madre fosse colei che, di fronte all'ordine di dividere fisicamente il bambino in due parti ed assegnarne una a ciascuna donna ${ }^{31}$, mossa da amore materno si sarebbe opposta alla divisione rinunciando

\footnotetext{
${ }^{25}$ Lo sviluppo di una teoria coerente delle presunzioni ha richiesto secoli di elaborazione dottrinale, analizzata da Motzenbäcker, 1958 e Gouron, 1990, 1992 (= 2000, III) e 1995(1) (=2000, VIII). Tale categoria è stata alle volte utilizzata per recuperare l'efficacia in giudizio di dichiarazioni testimoniali altrimenti inammissibili: cf. Gouron, 1995b (=2000, IX); Chiodi, 2017.

${ }^{26}$ Le classificazioni elaborate dalla dottrina di diritto canonico sul tema delle presunzioni sono state studiate nel dettaglio da Fiori: 2009 e 2013, pp. 428-446.

${ }^{27}$ Su cui v. Fiori, 2013, passim.

${ }^{28}$ J. Ph. Lévy, 1939, p. 109; Fiori, 2009, p. 92 e nt. 81.

${ }^{29}$ Niccolò Tedeschi ad X 2.23.08, de praesumptionibus, c. Quanto, n. 3, Commentaria super secunda parte secundi libri Decretalium (Torino 1577), fol. 118r; cfr. A. Fiori, 2009, pp. 78-79.

${ }^{30} \times 2.23 .2$, de presumptionibus.

${ }^{31}$ Diede l'ordine, Salomone, «non diffiniendo, sed potius tentando, causa inquirendi veri-
} 
alla propria ragione.

In una decretale accolta nello stesso titolo e relativa al caso di un sospetto eretico, la Literas vestras ${ }^{32}$, Innocenzo III aveva affermato che un sospetto, per quanto forte, non fosse mai sufficiente per condannare in un crimine grave quale quello di eresia. Ciò non toglie però che il sospetto vi fosse, e che dovessero essere presi provvedimenti: si optava in questo caso per la comminazione all'imputato di una penitenza, che valesse a chiarire se egli fosse un vero cattolico o piuttosto un finto converso. Solo in quest'ultimo caso lo si sarebbe potuto condannare per eresia.

Quale valore dare dunque alle presunzioni in giudizio? A Sinibaldo Fieschi non sfuggiva la scivolosità della materia. Insigne giurista, stava componendo il suo capolavoro, il commento alle Decretali di Gregorio IX, quando nel 1243 venne eletto al soglio pontificio con il nome di Innocenzo IV ${ }^{33}$. Commentando il c. Quia verisimile, Innocenzo tracciava un primo quadro generale sugli effetti delle presunzioni. Dopo aver respinto l'ammissibilità di ogni presunzione temeraria e accolto senza indugi il valore probatorio delle presunzioni violente, passava ad analizzare le diverse tipologie di praesumptio probabilis: essa poteva, di volta in volta, introdurre una prova semi-piena, o essere sufficiente ad indire una purgatio canonica, o ancora condurre direttamente a sentenza, come nel caso sottoposto all'attenzione di Salomone ${ }^{34}$. La pluralità di conseguenze era tale da rendere impossibile l'indicazione di una rigida disciplina che ne regolasse definitivamente I'uso. II Papa si limitava, dunque, a pronunciare una massima di prudenza: quan-

tatem, cuius filius esset. Nam non omnis vox iudicis auctoritatem continet rei iudicatae»: è quanto segnala nella glossa ordinaria (ad loc. cit.) Bernardo da Parma, dimostrando di aver ormai pienamente assimilato le dinamiche della novella natura inquisitoria del processo criminale.

${ }^{32}$ X 2.23.14, de praesumptionibus: «Propter solam suspicionem, quamvis utique vehementem, nolumus illum de tam gravi crimine condemnari, ... discretam ei poenitentiam iniungentes, ex qua valeat apparere, utrum in tenebris ambulet, an in luce, utrumve sit vere poenitens, an ficte conversus. Et si eum hoc modo verum catholicum cognoveritis, non sinatis ipsum indebite molestari; alioquin eum tanquam haereticum condemnetis». ${ }^{33}$ Paravicini Bagliani, 2004, p. 438. Per una biografia del Pontefice si consulti anche Melloni, 2013.

${ }^{34}$ Innocenzo IV, gl. Verisimile ad X 2.23.10, de praesumptionibus, c. Quia verisimile, Commentaria. Apparatus in V libros decretalium (Frankfurt a.M. 1570 - rist. Frankfurt a.M. 1968), fol. 281r: «Probabilis, quando semiplenam probationem inducit, infra de sen. excom. Inquisitionis (X 5.39.44), infra de resti. spo. Solicitae (X. 2.13.2). Quandoque sufficit ad inducendam purgationem, supra de purg. ca. Si quis de gradu (X 5.34.4), 2 q. 5 Presbyter (C. 2 q. 5 c. 5). Item quandoque requirit probationes contra se, ff. quod met. caus. Non est verisimile (D. 4.2.23), C. de contrahen. stip. Optimam (C. 8.37.14). Quandoque nullas admittit, nisi sit fama vel alia circunstantia contra eam, ut hic. Quandoque infundit probationem in reum, infra ut eccle. benefi. Ut nostrum (X 3.12.1), ubi no. C. de proba. Sive (C. 4.19.16). Quandoque etiam adducit secum sententiam, infra eodem Litteras (X 2.23.14), supra eodem Afferte (X 2.23.2), ff. de pact. I. Quod si servus (D. 2.14.21)». 
do si fosse proceduto sulla base di presunzioni, il giudice avrebbe dovuto multum temperare sententiam, soprattutto nel senso di non condannare, se non raramente e con moderazione, ad una pena pecuniaria o corporale.

Nota tamen quod ubi ex praesumptionibus proceditur multum debet iudex temperare sententiam, et maxime ne condemnet, nisi raro et modice ad poenam, nec pecuniariam, nec corporalem, quod potes bene colligere ex sententia Salomonis, supra eodem, Afferte (X 2.23.2), ubi licet ex praesumptione voluit adiudicari uni mulieri filium, non tamen voluit aliam condemnare ad mortem, ad quam iustissime erat condemnanda, si legitime esset probatum contra eam quod filium alterius clam surripuisset. ${ }^{35}$

L'espressione scelta da Sinibaldo, nisi raro et modice ad poenam nec pecuniariam nec corporalem, si presterà - come vedremo - ad una varietà di interpretazioni differenti e forzature più o meno evidenti. Nel Cinquecento Giulio Claro riconoscerà che i verba Innocentii venivano riportati dai Doctores "non multum fideliter" ${ }^{\prime \prime}$.

Con il precetto innocenziano tutti i successivi commentatori e trattatisti dovettero fare i conti. Esso, usato ancora lungo tutto il secolo successivo per conferire autorità alle concezioni teoriche che, contro le pressioni esercitate dalla prassi del foro, tendevano ad escludere l'inflizione di pene - specie se corporali - in assenza di prove piene, varrà invece ben presto a legittimare la possibilità per il giudice di pronunciare condanne definitive in presenza di sole presunzioni, purché venisse rispettato l'obbligo di mitigare la pena ordinaria.

Una ventina d'anni dopo l'enunciazione della doctrina Innocentii, nel commentare a sua volta il c. Quia verisimile, quello che è stato definito "il canonista più importante e brillante del secolo XIII"37, il cardinale Ostiense, per esporre il tema delle presunzioni si limitava a ricalcare il dettato del suo maestro ${ }^{38}$. Egli conclu-

${ }^{35} \mathrm{Ibid}$, Su questo passo, anche in relazione al commento al c. Nullus dubitat (X 2.23.4), cfr. Schmoeckel, 2000, pp. 332-334.

${ }^{36}$ Giulio Claro, Liber quintus receptarum sententiarum, § finalis, q. 20, n. 6, in Opera omnia (Venezia 1640), 248: «ubi ex praesumptionibus proceditur, multum debet iudex temperare suam sententiam, et maxime ne condemnet nisi raro, et modice ad poenam nec pecuniariam, nec corporalem. Et haec sunt verba Innocentii, licet non multum fideliter per Doctores relata».

${ }^{37}$ Pennington, 1993, p. 758.

${ }^{38}$ Ostiense ad X 2.23.10, de praesumptionibus, c. Quia verisimile, n. 3, In secundum decretalium librum commentaria (Venezia 1581 - rist. Torino 1965). Altrove, pronunciandosi sulla "pena per il sospetto", l'Ostiense l'aveva esclusa con fermezza muovendo da due princìpi contenuti nella compilazione giustinianea - quello tratto dalla I. Absentem del Digesto (D. 48.19.5), secondo il quale tutius est delinquentem absolvere, quam innocentem condemnare, e quello della I. Sciant del Codice (C. 4.19.25) che, come detto, richiedeva in criminalibus indiciis ad probationem indubitatis et luce clarioribus. Egli ricordava tuttavia che le presunzioni, a differenza del mero sospetto, erano in determinati casi suf- 
deva poi:

Hoc autem attendat iudex, quod ubi ex solis praesumptionibus ad diffinitivam procedit, non sit praeceps, nec nimis promptus ad reum condemnandum et maxime ad poenam corporalem infligendam. Nec etiam pecuniariam nisi modicam et perraro, ut sic semper sententiam moderetur. ${ }^{39}$

Pur nella sostanziale fedeltà all'originale formulazione del principio, mettendo a confronto le parole usate dall'Ostiense e quelle di Innocenzo si noterà che un lieve slittamento del centro d'equilibrio immaginato da Sinibaldo si era già verificato: pena corporale e pecuniaria non venivano più affiancate in posizione paritaria. Se l'una era da evitarsi ad ogni costo, per l'altra si potevano fare eccezioni, seppure, s'intende, raramente e con moderazione. L'interpretazione quasi impercettibilmente differente aprirà la via a una trasformazione del pensiero innocenziano che la dottrina successiva avrebbe costantemente ampliato.

In finale di secolo, mentre Guglielmo Durante riproponeva con fedeltà la massima innocenziana ${ }^{40}$, la strada aperta dall'Ostiense fu percorsa al di fuori del diritto canonico da Alberto Gandino ${ }^{41}$, che finì per offrire una lettura alternativa di tale precetto poi ampiamente seguito dalla dottrina successiva. Gandino infatti operò due distinzioni: la prima, tra cause civili e criminali; la seconda, tra pena pecuniaria e pene di altro tipo.

Nel titolo del suo celeberrimo Tractatus de maleficiis dedicato alle presunzioni ed agli indizi indubitati ${ }^{42}$ attraverso cui poteva pervenirsi a condanna, Gandino risolveva infatti la quaestio ${ }^{43}$ in discussione affermando che, se nelle cause civi-

ficienti per la condanna: «nec debet aliquis suspicionibus condemnari: quia tutius est delinquentem absolvere, quam innocentem condemnare, ff. de poenis Absentem, et in criminalibus probationes luce clariores exiguntur, C. de proba. Sciant ... sed et quandoque praesumptiones sufficiunt» (Summa aurea ad X 5.37, de poenis, § Quando sunt inferende, Venezia 1584, col. 1743).

${ }^{39} \mathrm{Ad}$ c. Quia verisimile supra cit., n. 4.

${ }^{40}$ Guglielmo Durante, Speculum iuris, II, 2, tit. De praesumptionibus, § Species, n. 6 (Lyon 1577), fol. 178r: «In summa nota, quod ubi iudex per praesumptionem procedit, valde sua debet sententiam temperare, et tardus debet esse ad poenam corporalem, vel pecuniariam infligendam... prout notatur, secundum Papam, Extra eodem Quia verisimile». ${ }^{41}$ La conoscenza dell'opera dell'Ostiense da parte di Gandino è nota: v. Fiori, 2013, p. 476. Per un profilo intellettuale e l'ampia bibliografia su Alberto Gandino si rinvia a Quaglioni, 2013.

${ }^{42}$ Alberto Gandino, Tractatus de maleficiis, in H. Kantorowicz, Albertus Gandinus und das Strafrecht der Scholastik II (Leipzig 1926), 90 ss.

${ }^{43}$ La quaestio (tit. de praesumptionibus et indiciis indubitatis, ex quibus condemnatio potest sequi, $\S 1$ ) riguardava il caso di un Tizio ucciso in una vigna, con Seio accusato del crimine. Nessuno tra i testimoni può affermare di aver visto Seio uccidere Tizio, ma essi depongono sulle seguenti circostanze: i due erano nemici; Seio è stato visto agitare una spada contro Tizio; in seguito egli è fuggito dal luogo in cui è stato commesso l'omicidio; 
li una sentenza definitiva poteva essere emanata sulla base di presunzioni iuris tantum, altrettanto non avrebbe potuto dirsi per quelle criminali ${ }^{44}$. La ratio che lo induceva ad effettuare tale distinzione di valore tra cause civili e criminali era indicata nel fatto che in queste ultime, poiché grande era il pericolo di un forte pregiudizio, sarebbe stato sempre opportuno usare la massima cautela ${ }^{45}$ : solo indizi indubitati avrebbero soddisfatto il requisito del massimo grado di chiarezza richiesto per la condanna in criminali ${ }^{46}$. Nella categoria degli indicia indubitata l'autore comprendeva esclusivamente una serie di prove tassativamente determinate, tra le quali la presunzione iuris et de iure, la confessione, la notorietà del fatto ${ }^{47}$. A chiudere la lista di indizi indubitati Gandino poneva tre esempi classici indicati da Tommaso da Piperata nel Tractatus de fama ${ }^{48}$ - la stanza con una sola uscita, il corpo nel vigneto, l'assassinio commissionato ${ }^{49}$-, ma solo per dissentire

infine, fama e voce pubblica affermano che sia Seio l'assassino. Ci si chiede: potranno questi indizi, letti congiuntamente, assurgere al rango di indicia indubitata e rendere dunque possibile procedere a sentenza? II caso, che diverrà di scuola, era stato posto da Tommaso da Piperata: cfr. infra, nt. 49.

${ }^{44}$ De maleficiis cit., 93: «Videtur quod ex predictis indiciis possit colligi tantum iuris presumptio contra predictum Seium ... Et quamvis in causa civili ex iuris tantum presumptione possit diffinitiva sequi sententia, ... tamen in criminali causa vel in causa questionis predicte ex tali presumptione iuris tantum non debebit vel poterit quis diffinitive damnari, quia in criminalibus causis intervenire debent et requiruntur probationes clariores luce». ${ }^{45}$ Ibid.: «in causis criminalibus perspicacius est consilium adhibendum, quia ibi maius periculum et detrimentum contingit». E ancora, ibid.: «ubi maius periculum vertitur, maior est requirenda cautela».

${ }^{46}$ Ibid., 94: "Sed licet ex ea sola probatione [rectius: praesumptione], que iuris est tantum, aliquis non possit vel debeat corporaliter diffinitive damnari, tamen ex ea sola presumptione, que iuris est et de iure, et ex quolibet alio indicio indubitato poterit contra aliquem diffinitiva formari sententia».

${ }^{47}$ Ibid., 95: "De quibus indicis indubitatis multa possunt notari exempla. Primum est indubitatum, quotiens contra aliquem est iuris et de iure presumptio ... Secundum est indicium indubitatum, quando constat quod aliquis in iudicio de crimine sponte sit confessus vel in tormentis et perseveraverit ... Quartum habetur indicium indubitatum, quotiens constat, quod crimen, quod adversus aliquem oritur, ita est publicum et manifestum, ut merito possit et debeat notorium appellari ...».

${ }^{48}$ L'opera fu composta nella seconda metà del Duecento, ed ebbe grande fortuna, a differenza del suo autore. Travolto nel conflitto bolognese che vedeva opporsi i guelfi capitanati dalla famiglia dei Geremei e i ghibellini stretti attorno ai Lambertazzi, per cui pubblicamente parteggiava, Tommaso fu forse vittima, in seguito al suo esilio, di una sorta di damnatio memoriae ufficiosa da parte dell'élite dei giuristi: cfr. Fraher, 1989, pp. 29-40. Sull'autore Padovani, 2013, pp. 1959 s. e la bibliografia ivi citata.

${ }^{49}$ L'autore, ponendosi in radicale antitesi con il nascente sistema di prova, aveva concepito tre casi ipotetici in cui l'evidenza della colpevolezza poteva dirsi certa, seppur a rigore non provata, e sostenuto dunque la validità di una condanna basata su soli indizi. I tre casi saranno destinati a far scuola, venendo riproposti - solo per nominare gli autori principali - da Giovanni d'Andrea (add. Sciant a Guglielmo Durante, Speculum iuris cit., II, 2, tit. De 
con le conclusioni del suo autore: propter talia vel similia non possit quis diffinitive in persona damnari. Quella adottata era una soluzione a suo avviso obbligata, perché condivisa da omnes sapientes, quos Bononie vidi et alibi, nonché osservata in via consuetudinaria ${ }^{50}$.

Consapevole che da un'applicazione rigorosa della propria tesi sarebbe derivata una sostanziale impossibilità di giungere a condanna, Gandino non mancò di segnalare a fine trattazione un'importante eccezione:

At si ex maleficio, ex quo essent talia indicia, deberet sequi pecuniaria pena, habeat [iudex] potestas arbitrium vel non, posset locum habere, quod scripsit dictus dominus Thomas. ${ }^{51}$

Che fosse o meno provvisto di arbitrium, si lasciava alla valutazione del giudice se giungere a sentenza in assenza di prove piene - pur trattandosi di materia criminale - fintantoché la pena prevista per il delitto commesso fosse stata solo pecuniaria. La modulazione della sentenza rimaneva dunque esclusa, e si imponeva l'assoluzione quando al delitto fosse collegata una pena corporale.

In un tentativo di aggirare il tradizionale rigore, Gandino offrì in questo modo una propria soluzione al problema della carenza di prove: relativamente fedele alla doctrina Innocentii, essa finì tuttavia per influenzarne I'interpretazione. Veniva confermata la corrispondenza tra qualità della prova e tipo di pena, e, pro-

probationibus, § Videndum, fol. 127r), Bartolo da Sassoferrato (ad D. 47.2.3, de furtis, I. Fur est manifestus, Commentaria, VI, Venezia 1526 - rist. Roma 1996, fol. 128v), Baldo degli Ubaldi (ad D. 28.5.92, De heredibus instituendis, I. Pactum eius, n. 9, In primam et secundam Infortiati partem commentaria, Venezia 1599, fol. 89v), Antonio da Budrio (ad X 2.19.5, de probationibus, c. Tertio loco, n. 20, Super secunda secundi Decretalium Commentarii, Venezia 1578, fol. 5r), Bartolomeo da Saliceto (ad C. 4.19.25, de probationibus, I. Sciant cuncti, n. 2, Lectura super novem libris Codicis, Lyon 1560, 289r: cfr. Garlati, 2004, pp. 406 s.) e Angelo Gambiglioni (De maleficiis tractatus, rubr. Comparverunt dicti inquisiti et negaverunt, n. 6, Venezia 1578, fol. 205r) oltre che dal Gandino. Per una loro lettura cfr. Tommaso da Piperata, Tractatus de fama, I, nn. 66-74, in Tractatus Universi luris XI, I (Venezia 1584), foll. 10r ss.

${ }^{50}$ De maleficiis cit., 98: «Ex predictis vero tribus indiciis ... scripsit dominus Thomas de Piperata posse ad condemnationem procedi, dicens sic intelligi I. ult. c. de probat. (C. 4.19.25) tamquam indubitatis indiciis, non obstante I. ff. de penis I. absentem, ubi ex presumptionibus nemo damnandus existit. Sed omnes sapientes, quos Bononie vidi et alibi, dicunt, et etiam vidi de consuetudine observari, quod propter talia vel similia non possit quis diffinitive in persona damnari». Massimo Vallerani ha ritenuto "non proprio congeniale" a Gandino il principio della maggior certezza delle prove nei processi criminali, accolto a suo parere solo in virtù del peso dell'opinione espressa dai dottori bolognesi e ribilanciato attraverso l'attribuzione al giudice di ampi poteri discrezionali nella valutazione degli elementi relativi alla persona e al reato: cf. Vallerani, 2005, pp. $45-47$ e 95-96, e 2009.

${ }^{51}$ lbid. 
seguendo sulla strada aperta qualche decennio prima dall'Ostiense ${ }^{52}$, si acuiva il distacco tra quelle che saranno le sorti di pena pecuniaria e corporale.

\section{Oscillazioni della dottrina intermedia: verso una communis opinio}

Riferimenti alla massima innocenziana si ritrovano nella letteratura, tanto civilistica quanto canonistica, lungo tutto il XIV secolo, sebbene di essa si continuino a dare interpretazioni difformi.

Se Bartolo, nel commentare la I. Sciant cuncti53, aveva soprasseduto sulla questione e Giovanni d'Andrea (in ciò seguendo l'esempio dell'Abbas antiquus ${ }^{54}$ ) si era limitato a recepire integralmente l'insegnamento di Innocenzo ${ }^{55}$, si intravede con Baldo un cambio di prospettiva:

Si [cum semiplena probatione] non adiungitur confessio, vel quia qualitas personae non patitur, vel quia cum hoc sit commissum arbitrio iudicis, non vult torquere, vel quia reus abest, vel quia statutum prohibet, tunc non potest imponi poena corporalis, quia propter inopiam probationis minuitur poena, hoc est, quia non potest plus punire in criminali quam condemnare in civili, haec enim est sententia Innocentii, quod ubi proceditur ex praesumptione imponatur minor poena. ${ }^{56}$

\footnotetext{
${ }^{52}$ V. supra, nt. 38.

${ }^{53}$ Bartolo ad C. 4.19.25, de probationibus, I. Sciant cuncti, Commentaria cit., VII, fol. 140v.

${ }^{54}$ Bernardo da Montmirat ad X 2.23.2, de praesumptionibus, c. Afferte, Lectura aurea super quinque libris Decretalium (Strasburgo 1510 - rist. Frankfurt a.M. 2008), fol. 118v: «Presumptio violenta sufficit ad sententiam, et nota hic: quod ubi ex presumptione iudicatur, multum debet iudex sententiam temperare ut non condemnet de facili ad penam corporalem, vel mulctam». Si noti che Innocenzo aveva classificato come probabilis la presunzione che qui viene definita violenta: v. supra, nt. 34.

${ }^{55}$ Giovanni d'Andrea ad X 2.23.2, de praesumptionibus, c. Afferte, n. 3, In secundum
} Decretalium librum Novella Commentaria (Venezia 1581 - rist. Torino 1963), fol. 177r: "Quando diffinitiva fertur ex praesumptione violenta temperat iudex sententiam, ut non de facili condemnet ad poenam corporalem, vel multam». Sulla condanna ex praesumptionibus l'autore si espresse anche nelle additiones allo Speculum di Guglielmo Durante, richiamando i già citati casi tipici di indizi indubitati per dissentire dal loro ideatore, Tommaso da Piperata, sostenendo che si trattasse di indizi sufficienti alla tortura, non ad una condanna: «Occisus est Titium in vinea, vel fundo; quod Seius occiderit, non sunt testes; sed sunt de inimicitia, de vibratione gladii contra illum, quod viderunt fugientem de loco maleficii tempore maleficii, item de voce et fama quod occidit: haec quatuor, secundum [Thomas de Piperata], bene probarent Titium per Seium occisum, et sic intelligit I. sciant. ... Potius videtur fienda tortura, ut ex ore suo iudicetur» (add. Sciant a Guglielmo Durante, Speculum iuris cit., fol. 127r). Tale passo verrà diversamente interpretato da Giovanni da Imola, che lo richiamerà a sostegno della possibilità di comminare pena corporale: v. infra, nt. 65.

${ }^{56}$ Baldo ad C. 4.19.25, de probationibus, I. Sciant cuncti, n. 15, In quartum et quintum Codicis libros commentaria (Venezia 1599), fol. 45v. Nel suo commento ai primi tre libri delle Decretales Baldo si era limitato a riportare le parole di Innocenzo, concludendo: «et 
Nell'escludere una pena corporale quando ad una prova imperfetta non si fosse aggiunta una confessione del reo, Baldo richiamava dunque l'insegnamento di Innocenzo come massima, per affermare che a causa della scarsità di prove la pena dovesse essere diminuita. In effetti, però, Innocenzo aveva sconsigliato al giudice di condannare, ogniqualvolta una praesumptio fosse stata determinante per il giudizio, e comunque di moderare la pena corporale o pecuniaria. Baldo invece, senza riproporre la raccomandazione ad evitare, quando possibile, la condanna, ne trasse due distinte regole: che non si potesse infliggere in nessun caso una pena corporale (come già aveva sostenuto Gandino), poiché si doveva sempre mitigare la sentenza propter inopiam probationis. Nulla sull'eventuale sanzione pecuniaria, che si confermava così liberamente applicabile.

In ambito canonistico Pietro d'Ancarano (che di Baldo era stato allievo nei suoi studi civilistici ${ }^{57}$ ) riaffermò il principio dell'impossibilità di condannare in base a prova presuntiva in un processo criminale, nel quale le probationes dovevano essere luce clariores, per conseguenza limitando la validità del principio innocenziano all'azione civile ex delicto.

Poco prima di giungere ad affermare, come di consueto, che quando si fosse proceduto per presunzioni il giudice doveva fortemente temperare la sentenza ${ }^{58}$, Pietro specificava infatti che quella di Salomone era stata una sentenza di condanna civile mirante alla restituzione, non criminale ${ }^{59}$. Applicando poi il concetto al caso dei presunti eretici, nel commento al c. Accusatus ribadiva che procedendo per presunzioni il giudice non avrebbe mai dovuto imporre una pena corporale, ma condannare civiliter:

Nota, quod quandoque de haeresi est quis vehementer suspectus: quae vehemens, et magna praesumptio non habetur pro plena probatione quo ad condemnationem ... nam si in aliis criminibus debent esse probationes luce clariores, multo fortius in isto crimine, ubi simul quis criminaliter damnatur, civiliter punitur, et eius posteritas perpetua notatur infamia ... Tamen circa hoc nota quod si iudex procedit quandoque ad sententiam ex praesumptionibus, numquam tamen debet imponere poenam corporalem, sed civiliter condemnare, quod patet ex sententia Salomonis, supra de praesumptionibus c. Afferte, et notat Innocentius eodem ti-

propter penuriam probationum [iudex] potest dispensari in minori pena» (Baldo ad $\mathrm{X}$ 2.23.10, de praesumptionibus, c. Quia verisimile, Ad tres priores libros decretalium commentaria, Torino 1578, 218v). La posizione di Baldo sarà richiamata da Angelo Gambiglioni: «recte locutus est Baldus in d. I. fi. C. de probationibus (C. 4.19.25), quod ubi iudex ex presumptionibus venit ad condemnationem est iusta causa minuendi pena» (De maleficiis tractatus cit., tit. Quod fama publica, n. 24, fol. 74r).

${ }^{57}$ Cfr. Cortese, Pennington, 2013, p. 1578.

${ }^{58} \mathrm{Nel}$ commento al c. Quia verisimile (X 2.23.10, de praesumptionibus), ibid., 267.

${ }^{59}$ Pietro d'Ancarano ad X 2.23.2, de praesumptionibus, c. Afferte, n. 3, Super Secundo Decretalium facundissima Commentaria (Bologna 1581), 265: "Nota quod ex falsa unica praesumptione iudex fert sententiam in causa, quia fuit violenta praesumptio. Et condemnavit in civili ad restitutionem, non criminaliter, mulierem, quae subtraxit». 
tulo c. Quia verisimile. ${ }^{60}$

Nell'escludere la condanna criminale a pena corporale, Pietro non faceva riferimenti al tipo di verdetto che sarebbe stato lecito ammettere in sede civile, agendo per un crimine. Nel caso di Salomone si era trattato di una condanna alla restituzione del bene sottratto. Ma si noti che, in conformità al pensiero innocenziano, s'era andata affermando la possibilità di agire civiliter de crimine mirando all'irrogazione di una pena pecuniaria, che non essendo pena vendicativa non era considerata pena criminale ${ }^{61}$ : nel silenzio dell'autore possiamo affermare, senza sbilanciarci, che ancora una volta il castigo fisico era l'unica conseguenza apertamente rigettata di una condanna priva di chiari riscontri probatori.

Concordi nel sostenere la legittimità della condanna presuntiva nelle sole cause civili si mostreranno anche Antonio da Budrio - che di Pietro era stato allievo prima e collega poi ${ }^{62}$ - e Francesco Zabarella ${ }^{63}$.

L'impostazione data alla materia da Antonio da Budrio veniva poi riproposta, letteralmente, da Giovanni da Imola, che alla presenza del maestro aveva conseguito il dottorato in diritto canonico nel $1402^{64}$. L'imolese segnalava, tuttavia,

${ }^{60}$ Pietro d'Ancarano ad VI 5.2.8, de haereticis, c. Accusatus, Super Sexto Decretalium acutissima Commentaria (Bologna 1583), 387 s. Sulla condanna, interamente fondata su presunzioni, dei sola suspicione notabiles - sospetti eretici ritenuti colpevoli in assenza di prove - ha scritto Fiori, 2016: cfr. in particolare p. 220 ss., sull'applicazione della doctrina Innocentii a tale categoria di soggetti.

${ }^{61}$ Innocenzo lo aveva affermato molto chiaramente nell'esegesi ad X 5.37.2, de poenis, c. Presbyteri (Commentaria cit., fol. 541v), e lo aveva ribadito nel Trecento Giovanni d'Andrea, nel commentare il medesimo canone, ad v. Solvatur, n. 1: "dicit Innocentius no. de crimine agi civiliter ad poenam pecuniariam, quia poena pecuniaria non est vindicta", In quintum Decretalium librum Novella Commentaria (Venezia 1581 - rist. Torino 1963), fol. 120r. Sul punto Fiori, 2020, p. 68.

${ }^{62}$ Condorelli, 2013a, p. 81. Antonio da Budrio ad X 2.23.2, de praesumptionibus, c. Afferte, n. 7, Super secunda secundi cit., fol. 68r: «diffinitiva quandoque profertur ex praesumptione violenta. Tamen ex ea non facile condemnat ad poenam corporalem, vel mulctam, ut patet hic: quia non fuit haec mulier iudicata ad mortem, vel mulctam, quod factum fuisset, si fuisset vere probatum eo quod alterius filium surripuisset, sed solum condemnata fuit ad restituitionem filii. ... Sed posset dici, quod hic ad hoc non agebatur, sed pro restitutione filii civiliter».

${ }^{63}$ Francesco Zabarella ad X 2.23.2, de praesumptionibus, c. Afferte, nn. 5-6, Super secundo Decretalium subtilissima Commentaria (Venezia 1602), fol. 64v: «ut eleganter dicit Innocentius in c. Quia verisimile (X 2.23.10), quando fertur sententia per praesumptionem, iudex debet [sententiam] mitigare: ne de facili imponere poenam vel mulctam, quod signa. Ex hoc dic, quod licet praesumptio hic sufficiat in causa ciuili, ut recideretur [sic] filius, non tamen ut pronunciaretur super crimine, ut imponeretur poena».

${ }^{64}$ Su Giovanni da Imola Padovani, 2017. Giovanni da Imola ad X 2.23.2, de praesumptionibus, c. Afferte, n. 4, In secundum decretalium commentaria (Venezia 1575), fol 139r: «Item nota quod licet diffinitiva quandoque proferatur ex presumptione violenta ad ef- 
che nonostante regulariter valesse il principio per cui una praesumptio violenta consentiva la condanna nelle cause civili ma non in quelle criminali, tuttavia un grado di violenza tale da non lasciare dubbi circa la colpevolezza dell'imputato avrebbe potuto giustificare l'inflizione di una pena corporale ${ }^{65}$. Si apriva in questo modo alla facoltà di infliggere una pena corporale, sinora unanimemente esclusa dai giuristi posteriori ad Innocenzo, e lo si faceva senza alcun riferimento alla mitigazione della pena.

La nascita di una soluzione interpretativa destinata ad assurgere a communis opinio $^{66}$, si dovette ad un altro allievo di Pietro d'Ancarano, Niccolò Tedeschi ${ }^{67}$. Nel commentare la classica sedes materiae, il Panormitano risolveva alcuni dubbi aperti dalla dottrina precedente attraverso una trattazione estremamente chiara e completa. Come sappiamo, v'era chi reputava che l'episodio da cui era scaturita la famosa sentenza di Salomone vedesse la donna agire per ottenere non una condanna criminale, ma la restituzione - civile - del figlio. L'Abate credeva tuttavia che tale distinzione fosse irrilevante: o si agiva civilmente, e dunque non era inflitta alcuna pena, sia che si arrivasse a sentenza in base a presunzioni che in base a prove legali; oppure lo si faceva penalmente (criminaliter). In tal caso,

fectum civilem, tamen ex illa non faciliter quis condemnatur ad penam corporalem vel mulctam, ut hic patet: quia non fuit haec mulier, quae habebat penes se infantem vivum iudicata ad mortem vel mulctam, quod factum fuisset si vere fuisset probatum quod alterius filius subripuisset. Facit quod habetur in I. i C. ad I. Faviam de plagia (C. 9.20.1). Sed solum condemnata fuit ad restitutionem filii. Facit quod no. gl. infra de purgatione canonica c. Inter (X 5.34.10) in ultima gl. et Innocentius infra eodem Quia verisimile (X 2.23.10). Posset tamen dici quod ad hoc non fuit actum nisi pro restitutione filii civiliter». ${ }^{65}$ Ibid., n. 6: «Adverte tamen, quia ut tetigi in notabilibus, licet praesumptio violenta sit sufficiens ad condemnationem in civili non tamen videtur sufficiens in criminali ut dixi, in notabilibus. Quod est verum regulariter per I. Absentem ff. de poenis (D. 48.19.5), fallit secundum lohannem Andreae si praesumptio est adeo violenta, quod omnino videatur inferre maleficium, puta si aliquis repertus est occisus in camera, quae non habet nisi unum ostium, et visus est unus exire palido vultu fugiens cum ense sanguinolento, nam contra talem videtur posse veniri ad poenam corporalem, ut ipse notat in Spec., de probationibus, $\S$ finalis super versiculo xiii, et ibi etiam aliqua alia exempla ponit». La possibilità di comminare poena corporalis è dedotta da quanto affermato da Giovanni d'Andrea nelle additiones allo Speculum di Guglielmo Durante. Si ricorderà, però, che Giovanni d'Andrea aveva piuttosto sostenuto il contrario, ritenendo indizi di tal genere validi per l'inflizione della tortura, non della condanna: v. supra, nt. 55.

${ }^{66}$ Tale è ritenuta da Bartolomeo Bellencini, Felino Sandei, Alfonso Vilagut e Ippolito de' Marsilii: cfr. Fiori, 2016, p. 221 e nt. 142, e Alessi, 1979, p. 69, nt. 69.

${ }^{67}$ Discendente da famiglia di origine tedesca, allievo, oltre che di Pietro d'Ancarano a Bologna, del cardinale Zabarella a Padova, Niccolò diverrà abate dell'abbazia di Maniace (1425), nella diocesi di Messina, e in seguito arcivescovo di Palermo (1434): di qui gli appellativi di Abbas Siculus (o modernus, per distinguerlo dall'Abbas antiquus Bernardo da Montmirat) e Panormitanus. Cfr. la voce di Condorelli, 2013b, alla quale si rimanda anche per la bibliografia sull'autore. 
quando il delitto risultasse provato in base a sole presunzioni, v'era da prestare attenzione al valore di esse: in presenza di presunzioni violente, tali da permettere di pronunciare una regolare sentenza civile, nell'azione criminale il giudice avrebbe potuto valutare liberamente la loro forza al fine di modulare la sanzione, multando o infliggendo pene di altro genere; se invece esse fossero state urgentissime, poteva essere inflitta la pena ordinaria, anche massima ${ }^{68}$. È, questa, un'ulteriore derivazione originata dalla riflessione canonistica: su tale potere-dovere in capo al giudice di infliggere la pena ordinaria quando convinto da presunzioni tanto persuasive da non lasciare spazio ad una diversa ricostruzione dei fatti concorderanno giuristi in utroque del calibro di Francesco Accolti da Arezzo ${ }^{69} \mathrm{e}$ Filippo Decio $^{70}$.

Niccolò Tedeschi proseguiva, poi, esplicitamente limitando l'ambito di applicabilità della soluzione proposta da Innocenzo IV $^{71}$ alle sole praesumptiones hominis. Si escludeva quindi che vi potesse essere qualsiasi mitigazione di pena qualora la presunzione fosse posta dalla legge: non più solo iuris et de iure, il qual caso non aveva mai posto interrogativi, ma anche iuris tantum ${ }^{72}$.

\footnotetext{
${ }^{68}$ Niccolò Tedeschi ad X 2.23.2, de praesumptionibus, c. Afferte, n. 6, Commentaria super secunda parte secundi libri Decretalium cit., fol. 115v: «Dic tu, quod aut agitur civiliter, et regulariter non infligitur poena, sive procedatur ad sententiam ex praesumptionibus, sive ex aliis legitimis probationibus ... Aut agitur criminaliter et constat de delicto ex praesumptionibus dumtaxtat: ... si enim sunt urgentissimae, tunc potest infligi poena ordinaria quantuncunque maxima. ... Aut praesumptiones non sunt urgentissimae, tamen sunt in tantum violentae, quod potest ferri sententia civiliter: et tunc habet locum iste textus et in summa secundum earum validitatem potest iudex exasperare sententiam mulctando, vel aliam poenam infligendo».
}

${ }^{69}$ Francesco Accolti ad X 2.19.5, de probationibus, c. Tertio loco, n. 17, In primi, secundi et quinti Decretalium titulos commentaria (Venezia 1581), fol. 57v: «Non recedo a communi sententia, sed dico declarando ipsam, quod iudex potest condemnare ex indiciis indubitatis, quando sunt talia quae inducunt quasi notorium praesumptionis, vel manifeste ponunt factum ante oculos».

${ }^{70}$ Filippo Decio ad X 2.19.5, de probationibus, c. Tertio loco, n. 60, In Decretalium Volumen perspicua Commentaria (Venezia 1576), fol. 201v: «in praesumptionibus indubitatis dicendum videtur, quod si adeo sunt violentae, quod aliter iudex credere non possit, quod tunc poena ordinaria imponatur».

${ }^{71}$ Fedelmente riportata dall'Abate nel commento ad X 2.26.15, de praescriptionibus, c. Auditis, n. 32, Super tertia parte secundi Decretalium libri (Torino 1577), fol. 36r: «no. Innocentius in c. quia verisimile, de praesumptionibus, ubi dicit notabiliter quod quando iudex procedit super delicto ex praesumptione debet multum temperare sententiam suam, maxime ne condemnet nisi raro et modice ad poenam corporalem vel ad pecuniariam». ${ }^{72}$ Niccolò Tedeschi ad X 5.1.23, De accusationibus, inquisitionibus et denunciationibus, c. Accedens, n. 6, Commentaria ad quartum et quintum libros Decretalium (Torino 1577), fol. 71v: «iudex debet temperare sententiam suam, quando procedit ex praesumptione hominis, non autem ex praesumptione iuris». Gandino, al contrario, era stato netto nel negare alle presunzioni iuris tantum il valore di probationes luce clariores sufficienti a 
I medesimi concetti saranno espressi da Felino Sandei, che a fine XV secolo ultimava il suo commento alle Decretali. Analizzando il c. Afferte informava innanzitutto che ubi proceditur ex praesumptionibus ista est iusta causa minuendi poenam. La portata dell'affermazione era tuttavia precisata subito dopo attraverso il ricorso a quattro limitazioni ${ }^{73}$. La massima non avrebbe trovato applicazione, e il giudice avrebbe quindi dovuto infliggere la pena ordinaria, quando la presunzione fosse approvata dalla legge stessa in materia criminale, poiché sulla base di una presunzione del genere si poteva arrivare a condanna in ogni causa, anche capitale; quando si agisse per l'imposizione di una pena pecuniaria o corporale non grave, come poteva essere la fustigazione, non essendovi in questo caso la necessità di mitigare ulteriormente il castigo; quando si procedesse per un caso di omicidio sulla base di probabilia $\operatorname{argumenta}^{74}$; quando, infine, il giudice fosse mosso a sentenza da una presunzione violentissima.

\section{Oltre la scienza canonistica: la fortuna della doctrina Innocentii tra giurisprudenza consiliare e pratiche criminali}

Abbiamo sinora seguito le vie per cui i canonisti seppero giustificare condanne

condanna: v. supra, nt. 46.

${ }^{73}$ Felino Sandei ad X 2.23.2, de praesumptionibus, c. Afferte, nn. 6 ss., Secunda in quinque Decretalium libros pars (Lyon 1549), fol. 220r: "Sed ista limita primo, nisi iudex moveret ex praesumptione quam lex expressa approbaret in criminibus, quia ex illa potest condemnare in omni causa etiam capitali ... Secundo limita, nisi agatur de imponenda poena poecuniaria vel corporali, non gravi, puta usque ad fustigationem quia tunc praesumptio vehemens sufficit ad condemnationem: maxime, si est approbata a iure generaliter, licet non in criminalibus ... Tertio limita non procedere in casu cap. Pro humani De homicidio in Sexto (VI 5.4.1) ... Quarto limita nisi iudex moveretur ex praesumptione violentissima ... Et hoc modo intelligitur I. sciant cuncti, in verbo indubitatis indiciis». Su Felino Sandei Di Renzo Villata, 2000.

${ }^{74}$ Il caso è interessante e merita un approfondimento. La terza limitazione si riferisce all'omicidio commissionato, di cui tratta il capitolo Pro humani, tit. de homicidio del Liber Sextus (VI 5.4.1), il cui testo avrebbe potuto dare man forte ai detrattori della doctrina Innocentii. Esso, nella parte finale, recita: «Et postquam probabilibus constiterit argumentis aliquem scelus tam execrabile commisisse, nullatenus alia excommunicationis, vel depositionis, seu diffidationis adversus eum sententia requiratur». Cosa individuare in quei probabilia argumenta? Filippo Decio aveva sostenuto la possibilità di condannare sulla base di semplici probationes per argumenta (Consilia sive Responsa, Lyon 1546, cons. 189, n. 4, fol. 138v), ma Felino così spiegava il suo dissenso: «sed oportet dicere, quod [textus c. pro humani] sit speciale in odium illius criminis, sicut etiam est speciale in crimine falsi, ut suspicio pro falsitate habeatur ... Posset etiam dici, quod Innocentius non dicit quod non sit condemnandus quis: sed dicit quod non de facili. Vel posset dici, quod verbum probabilibus intelligitur id est indubitatis, prout in sequenti limitatione» (ibid., $\mathrm{n}$. 9). Anche per un crimine esecrabile quale l'assassinio, dunque, rimaneva possibile infliggere la pena ordinaria solo quando si disponesse di indicia indubitata. 
basate su prove presuntive, legittimando una prassi teoricamente inammissibile non solo per il diritto giustinianeo, ma anche per la prima elaborazione canonistica: damnari non valet nisi aut convictus aut sponte confessus, stabiliva il Decreto di Graziano ${ }^{75}$.

La fortuna della dottrina innocenziana però - come abbiamo visto - scavalcò presto i confini della scientia iuris canonistica per essere definitivamente accolta dal mondo dei legisti.

Dalla prima metà del Quattrocento la massima aveva attirato l'attenzione dei giuristi consulenti. Di fronte all'impossibilità di ottenere una sentenza assolutoria, il precetto innocenziano tornava utile nel fornire un mezzo per sottrarre l'imputato a pene più severe. Esso d'altra parte ben si conciliava anche con il rigore di Gandino, che, ammettendo la sentenza per presunzioni nei soli crimini puniti con pena pecuniaria, si poneva come irrinunciabile punto di riferimento ove fosse necessario valorizzare le esigenze di garanzia del reo ed evitare la condanna ${ }^{76}$. E così, ogni volta che una probatio plena non potesse dirsi raggiunta, non era infrequente vedere le posizioni dei giuristi oscillare tra l'uno e l'altro principio: l'assoluta impossibilità di condannare e la necessità di mitigare la pena furono sostenute a fasi alterne, sulla base delle circostanze del caso. Alle volte anche all'interno del medesimo consilium, ben potendo la richiesta di condanna a pena moderata essere posta in via subordinata al mancato accoglimento della domanda assolutoria.

In tal senso si espresse, ad esempio, Bartolomeo Cipolla in uno dei suoi consilia. L'imputato, un certo Parmesanello, era accusato di aver ucciso un uomo durante una rissa. Le prove gravanti su di lui erano obscurae et suspectae, essendovi da un lato un certo numero di testimoni singulares e contraddittori, dall'altro una serie di indizi urgentissimi. Sottoposto ai tormenti, per più giorni di fila egli aveva

\footnotetext{
${ }^{75}$ C. 2 q. 1. c. 1.

${ }^{76}$ In questo senso Paolo di Castro, l'allievo di Baldo da più parti considerato il maggior giurista della sua epoca (Cortese, 2013, p. 1507 e D’Amelio, 1979, p. 531). Chiamato in causa nel giudizio contro la moglie di tale Francesco da Imola, morto per avvelenamento nel settembre 1402, Paolo si appellava all'autorità di Gandino per sostenere l'assoluzione della donna. Gravata da indizi più che convincenti, contro di lei non si era tuttavia formata piena prova, e così il castrense ammoniva: «Gandinus in opere suo solemni rubrica ... dicit quod omnes doctores qui erant Bononiae tempore suo dixerunt et ita etiam vidit ubicumque practicari quod talia indicia sic urgentia non sufficiunt ad condemnationem poenae corporalis, sed dumtaxat pecuniariae. Et propter eius dictum non est diu quod quendam defendi a poena capitali contra quem erant ita fortia et indubitata indicia quod animus et intellectus non poterat credere quod alius fecisset homicidium quam ipse. Concludendum est ergo in tutiorem et benigniorem partem sententiam absolutoriam ... sed si erat tale maleficium de quo imponebatur poena pecuniaria, bene potest fieri condemnatio» (Consilia et allegationes. Ed: Bartholomaeus Cepolla, Nürnberg 1495, cons. 197, fol. 166v; si noti che il consilium è numerato diversamente a seconda delle edizioni consultate: in Id., Consiliorum sive responsorum, II, Venezia 1581, è il n. 299, fol. 147r).
} 
superato la tortura senza confessare, ma v'era il dubbio che gli elementi di prova a carico dell'imputato non fossero stati per tale via del tutto purgati. Anche ammettendo tale circostanza, concludeva Cipolla dopo aver richiamato la doctrina Innocentii e il dictum Gandini,

ex indiciis quantuncunque urgentibus quis non debet ad mortem et ad poenam corporalem condemnari, sed poena debet minui et in poenam tantum pecuniariam condemnari ... Et ideo ex predictis concludo quod ille Parmesanellus non potuit de iure condemnari ad mortem, nec ad aliquem poenam corporalem, sed debet absolvi vel condemnari poena pecuniaria vel aliqua simili. ${ }^{77}$

La più ampia diffusione del principio ubi proceditur ex praesumptionibus minuitur poena derivò però dalla sua completa assimilazione da parte della criminalistica cinquecentesca. Practicae criminales $^{78}$, trattati ${ }^{79}$ e decisioni dei supremi

${ }^{77}$ Consilia criminalia (Venezia 1575), cons. 32 (inc. Quidam rustici), nn. 20-21, foll. 133r-v. Anche nel cons. 6 (inc, Veritas primi dubii), sulla scorta degli insegnamenti di Innocenzo e Gandino, Cipolla argomentava a favore di un accusato di omicidio: «cum igitur dictus Baptista tortus fuerit et non confessus, nec existerint vere probationes, potius debeat absolvi in totum vel saltim non debeat pati poenam corporalem vel pecuniariam» (ibid, n. 12 , foll. $24 \mathrm{v}-25$ r). Altrove, in presenza di un grado probatorio di più salda tenuta, l'autore allegava la doctrina Innocentii per evitare all'imputato la pena di morte, accettando una minor poena corporale che non può certo definirsi modica: cfr. ibid., cons. 27, nn. 13 e 16, foll. 107v-108r, ove Bartolomeo conclude per l'amputazione della mano destra in aggiunta al bando. Per la mitigazione della pena si esprimono anche: Alessandro Tartagni, Consiliorum seu responsorum (Venezia 1610), I, cons. 15, n. 3, fol. 24v; III, cons. 115, n. 8, fol. 102r; VII, cons. 188, n. 12, fol 141r; Andrea Barbazza, Consiliorum sive responsorum, cons. 62 , n. 4 (Venezia 1581), fol. 195r.

${ }^{78}$ Le note che seguono vogliono offrire, senza pretese di esaustività, una panoramica sull'ampio utilizzo del principio innocenziano da parte della dottrina criminalistica: cfr. Ippolito Marsili, Practica causarum criminalium, quae Averolda nuncupatur, Lione 1551, c. Postquam, n. 50, fol. 14r; Giulio Claro, Liber quintus receptarum sententiarum, $\S$ finalis, q. 20, n. 6, in Opera omnia (Venezia 1640), 248; Pietro Follerio, Practica criminalis, § Poenis debitis feriantur, n. 59 (Venezia 1558), 402; Prospero Farinacci, Praxis et Theoricae Criminalis, I, 2 (Lyon 1614), q. 52, n. 66, 367; Benedict Carpzov, Practica nova, III, q. 116, n. 57 (Wittenberg 1684), 149. Sul genere delle Practicae Garlati, 2016. All'attività criminalistica di Pietro Follerio ha dedicato i suoi studi Miletti, 2015.

${ }^{79}$ Giacomo Menochio, De Praesumptionibus, I (Venezia 1587), q. 97, nn. 23 ss., fol. 75v. Con Tiberio Deciani il principio viene applicato alla prova del dolo: «Haec autem divisio eo tendit, ut sciamus dolum verum magis ac gravius puniri, quam praesumptum» (Tractatus criminalis, I, IV, n. 17, Torino 1593, fol. 5r). Juan López de Palacios Rubios (allegatio $\S 11$, in Ambrogio Vignati, Elegans ac vtilis tractatus de haeresi, Roma 1581, foll. 108v ss.) e Diego da Simancas (Institutiones Catholicae, Valladolid 1552, cap. 49, n. 14, foll. $185 \mathrm{v}-186 \mathrm{r}$ ) applicarono il principio ai sospetti eretici, categoria per la quale a rigore l'esistenza di una presunzione di legge avrebbe dovuto escludere la possibilità di inflizione di sanzioni arbitrarie: cfr. in proposito Fiori, 2016, p. 223. Una limpida ricognizione dello stato dell'arte in materia di condanna ex presumptionibus è effettuata da Egidio Bossi 
tribunali d'Europa ${ }^{80}$ fecero proprie le parole di Innocenzo, ormai definitivamente recependole nella formula secondo cui, in caso di condanna per presunzioni, numquam debet imponi vera pena delicti sed alia mitior ${ }^{81}$. Tesero talvolta ad avvalorarle attraverso il ricorso ad auctoritates ancor più risalenti ${ }^{82}$, ne soppesarono le interpretazioni che la dottrina canonistica aveva fornito, per dare infine generale accoglimento all'impostazione elaborata dal Panormitano: ammessa la facoltà di arrivare a sentenza in materia criminale, condannare a pena ordinaria era legittimo, in presenza di presunzioni ed indizi lege approbatis; modulare la pena era dovuto, procedendo per praesumptiones hominis ${ }^{83}$.

(Tractatus varii, Lyon 1562, tit. de convictis, nn. 34 ss., 154ss): circa la possibilità di avvalersi per la prova del delitto, in Bossi, di indizi e presunzioni ha scritto Di Renzo Villata, 1996, pp. 461-463.

${ }^{80}$ Nicola Boerio, Decisiones Aureae (Lyon 1544), dec. 164, n. 6, fol. 175r.

${ }^{81}$ L'espressione, di Ippolito Marsili (loc. supra cit., nt. 78, e Grassea. Commentaria super titulis ff. ad legem corneliam de sicariis et ad legem pompeam de parricidiis et ad legem corneliam de falsis, ad. I. Cornelia de sicariis, § Divus Adrianus, n. 25, Lyon 1538, fol. 21r), venne impiegata con lievissime alterazioni da tutti gli autori richiamati nelle precedenti note: «non debet puniri vera pena delicti sed alia mitiori arbitrio iudicantis» è ad esempio la formula utilizzata dal Boerio (loc. supra cit., nt. 80), «numquam ei imponitur poena ordinaria, et vera poena delicti, sed alia mitior» le parole di Pietro Follerio (loc. supra cit., nt. 78).

82 È fenomeno, se non ignoto, di certo infrequente nella dottrina precedente. Ippolito de' Marsili (loc. supra cit.), nel concordare con Innocenzo che in caso di condanna ex praesumptionibus non si sarebbe mai dovuto imporre la vera poena delicti, sed alia minor, poneva l'affermazione in diretta correlazione - quasi ne fosse una conseguenza - con un canone grazianeo (C. 2 q. 1 c. 20) il quale sosteneva fermamente l'impossibilità di condannare prima che contro il sospettato fosse stata raggiunta una veram iustamque probationem. Ciò segnala al contempo la piena accettazione della facoltà di punire, seppur mitigando il castigo, in assenza di reali prove e l'ansia di inserire la condanna presuntiva in un quadro ordinato e coerente in ogni suo punto: da Graziano, alla dottrina intermedia, per giungere senza strappi evidenti alla pratica cinquecentesca. La medesima esigenza dovette avvertire André Tiraqueau, che nella ventisettesima causa del trattato De poenis temperandis aut remittendis (causa $27, \mathrm{n} .3$, Lyon 1559,108 ) inserisce, a sostegno della tesi per cui cum quis condemnatur non ex evidentibus probationibus sed ex vehementi praesumptione ... levius certe est puniendus, un riferimento alla lex Lombarda: in essa una norma (Lomb. 1.9.35, tit. De homicidio liberorum hominum, I. Qui vero intra treuguam) stabiliva, infatti, che quando un omicidio fosse stato provato attraverso il duello - che non rappresentava certo una prova vera et indubitata - lo sconfitto non sarebbe stato condannato a morte, ma solo all'amputazione della mano. II richiamo al duello era peraltro già presente in Baldo (ad C. 4.19.25, de probationibus, I. Sciant cuncti, nn. 18-19): cfr. Schmoeckel, 2000, p. 282.

${ }^{83}$ Tommaso Grammatico, Decisiones, dec. 42, n. 1 (Venezia 1551), fol. 54v: «ubi non ex probationibus, sed ex praesumptionibus etiam violentis proceditur contra delinquentem seu inquisitum, non potest inquisitus ipse ad mortem condemnari, sed iusta est causa in eo casu minorandi poena ... et extantibus tot praesumptionibus et indiciis lege approba- 
Alcuni aspetti della condanna presuntiva rimasero tuttavia ben più controversi. Lo riferiva, all'alba del XVII secolo, Prospero Farinacci, accingendosi ad affrontare un'ampia trattazione su questa materia multum intricata, a suo parere a doctoribus non perfecte tractata, et a iudicantibus nostro tempore non bene forsan intellecta ${ }^{84}$. Dibattuta rimase innanzitutto la questione della pena corporale, su cui non si formò un accordo tra doctores, più inclini ad escluderla, e prassi dei tribunali supremi - Senato e Sacro Regio Consiglio in primis ${ }^{85}$-, che correntemente la applicavano. II giureconsulto romano, se da un lato si professava fermamente convinto della necessità di escludere l'imposizione di pene corporali in assenza di pienezza probatoria, finiva tuttavia per ammettere e quasi consigliare ai giudici la condanna, oltre che all'esilio, al tratto di corda (l'ictus funis, consistente nel legare i polsi del reo dietro la schiena e poi nell'issarne il corpo con una carrucola) ove persuasi da indizi e presunzioni violente ${ }^{86}$ : una pena corporale mite, in sostanza, come prospettato quasi quattro secoli addietro da Innocenzo IV. Parimenti discussa rimarrà la questione della condanna a pena ordinaria in presenza di praesumptiones urgentissima $e^{87}$, che, sostenuta tra gli altri da Niccolò Tedeschi, Felino Sandei, Francesco Aretino e Filippo Decio, aveva rappresentato un rilevante approdo della dottrina canonistica.

tis in omnis causa etiam capitali iudex condemnare potest, ut tenet Abb. in c. Accedens ii col. ver. nec obstat». Analogamente si espressero Bartolomeo Bellencini (ad X 2.23.2, de praesumptionibus, c. Afferte, Apostillae super domini Abbatis necnon domini Antonii de Butrio Lecturas, Venezia 1477, fol. 63v-64r), Antonio Gómez (Variae resolutiones iuris civilis, communis et regii, III, c. 12, n. 25, Frankfurt a.M. 1597, p. 531), oltre al già citato Felino Sandei (v. supra, nt. 73).

${ }^{84}$ Prospero Farinacci, Praxis et Theoricae Criminalis, III (Lyon 1616), q. 86, 79r. La quaestio, che in oltre ventiquattro colonne affronta l'argomento "an et quando ex delicti praesumptionibus possit quis condemnari", rappresenta forse la più completa trattazione sul tema (sebbene non possa dirsi la più lineare, densa com'è di ampliationes, limitationes e sublimitationes alla regola generale, corredate a loro volta da una straordinaria profusione di auctoritates pro e contra). Sull'autore Mazzacane 2013.

${ }^{85}$ Ce ne dà notizia Giulio Claro (Liber quintus receptarum sententiarum, § finalis, q. 20, n. 6, in Opera omnia (Venezia 1640), 249: «quotidie ex indiciis et praesumptionibus rei per Senatum condemnantur, non quidem in poenam mortis, neque poneam ordinariam ipsius delicti, sed in poenas etiam corporales, puta triremium, fustigationis, ictuum funis ... Et idem servatur in Sacro Consilio Neapolitano». Le prassi dei grandi tribunali (in particolare quelli muniti della facoltà di giudicare secundum conscientiam) influenzarono notevolmente le opinioni dei giuristi che attorno a ciascuno di essi gravitavano. Tra gli studi che hanno approfondito il ruolo dell'arbitrium e della pena straordinaria nella giustizia criminale d'età moderna si può vedere (oltre ai testi cit. supra, nt. 5), il fondamentale saggio, relativo all'area lombarda, di Cavanna, 1999 (=2007, pp. 1037-1068).

${ }^{86}$ Op. supra cit., nn. 78-83, p. 86, e nn. 113 ss., pp. 89-90 (in particolare n. 117, su exilium e ictus funis).

${ }^{87}$ Le auctoritates a favore e contrarie sono ancora una volta riportate dal Farinacci: loc. supra cit., nn. 110-111, pp. 88-89. 
Il dibattito attorno alla condanna per presunzioni dunque seguirà, per vie che non è qui più possibile indagare. Il principio le cui vicende abbiamo cercato di esaminare, nato come raccomandazione di prudenza ai giudici, si è rivelato uno strumento capace di incidere sulla teorica rigidità del sistema di prova legale, ammettendo discrezionalità nel modulare la pena e nell'applicarla anche laddove non vi fosse pienezza probatoria. Il lavorio dottrinale progressivamente sviluppatosi a partire dalla doctrina Innocentii ha sortito i suoi effetti ben al di là dell'ambiente canonistico nel quale si è per la massima parte svolto, venendo infine recepito in ogni ambito dell'universo criminalistico: anche per tale via si manifesta il ruolo centrale assunto dalla scienza canonistica nella riflessione dottrinale sulla teoria della prova legale.

\section{Fonti}

Alberto Gandino: Tractatus de maleficiis, in Kantorowicz H., Albertus Gandinus und das Strafrecht der Scholastik, II, Leipzig, 1926

Alessandro Tartagni: Consiliorum seu responsorum, Venezia, 1610

Andrea Barbazza: Consiliorum sive responsorum, Venezia, 1581

André Tiraqueau: De poenis temperandis aut remittendis, Lyon, 1559

Angelo Gambiglioni: De maleficiis tractatus, Venezia, 1578

Antonio da Budrio: Super secunda secundi Decretalium Commentarii, Venezia, 1578

Antonio Gómez: Variae resolutiones iuris civilis, communis et regii, Frankfurt a.M., 1597

Baldo degli Ubaldi: In primam et secundam Infortiati partem commentaria, Venezia, 1599

Baldo degli Ubaldi: In quartum et quintum Codicis libros commentaria, Venezia, 1599

Baldo degli Ubaldi: Ad tres priores libros decretalium commentaria, Torino, 1578

Bartolo da Sassoferrato: Commentaria, Venezia 1526, rist. Roma, 1996

Bartolomeo Cipolla: Consilia criminalia, Venezia, 1575

Bartolomeo Bellencini: Apostillae super domini Abbatis necnon domini Antonii de Butrio Lecturas, Venezia, 1477

Bartolomeo da Saliceto: Lectura super novem libris Codicis, Lyon, 1560

Benedict Carpzov: Practica nova, Wittenberg, 1684

Bernardo da Montmirat: Lectura aurea super quinque libris Decretalium, Strasburgo, 1510, rist. Frankfurt a.M., 2008

Diego da Simancas: Institutiones Catholicae, Valladolid, 1552 
Egidio Bossi: Tractatus varii, Lyon, 1562

Felino Sandei: Secunda in quinque Decretalium libros pars, Lyon, 1549

Filippo Decio: In Decretalium Volumen perspicua Commentaria, Venezia, 1576

Francesco Accolti: In primi, secundi et quinti Decretalium titulos commentaria, Venezia, 1581

Francesco Zabarella: Super secundo Decretalium subtilissima Commentaria, Venezia, 1602

Giacomo Menochio: De Praesumptionibus, I, Venezia, 1587

Giovanni da Imola: In secundum decretalium commentaria, Venezia, 1575

Giovanni d'Andrea: In secundum Decretalium librum Novella Commentaria, Venezia, 1581, rist. Torino, 1963

Giovanni d'Andrea: In quintum Decretalium librum Novella Commentaria, Venezia, 1581, rist. Torino, 1963

Giulio Claro: Liber quintus receptarum sententiarum, in Opera omnia, Venezia, 1640

Guglielmo Durante: Speculum iuris, Lyon, 1577

Innocenzo IV: Commentaria. Apparatus in V libros decretalium, Frankfurt a.M., 1570, rist. Frankfurt a.M., 1968

Ippolito Marsili: Practica causarum criminalium, quae Averolda nuncupatur, Lyon, 1551

Ippolito Marsili: Grassea. Commentaria super titulis ff. ad legem corneliam de sicariis et ad legem pompeam de parricidiis et ad legem corneliam de falsis, Lyon, 1538

Juan López de Palacios Rubios: Elegans ac vtilis tractatus de haeresi, Roma, 1581

Niccolò Tedeschi: Commentaria super secunda parte secundi libri Decretalium, Torino, 1577

Niccolò Tedeschi: Super tertia parte secundi Decretalium libri, Torino, 1577

Niccolò Tedeschi: Commentaria ad quartum et quintum libros Decretalium, Torino, 1577

Nicola Boerio: Decisiones Aureae, Lyon, 1544

Ostiense: In secundum decretalium librum commentaria, Venezia, 1581, rist. Torino, 1965

Ostiense: Summa aurea, Venezia, 1584

Paolo di Castro: Consilia et allegationes. Ed: Bartholomaeus Cepolla, Nürnberg, 1495

Pietro d'Ancarano: Super Secundo Decretalium facundissima Commentaria, Bologna, 1581 
Pietro Follerio: Practica criminalis, Venezia, 1558

Pietro d'Ancarano: Super Sexto Decretalium acutissima Commentaria, Bologna, 1583

Prospero Farinacci: Praxis et Theoricae Criminalis, Lyon, 1614-1616

Tommaso da Piperata, Tractatus de fama, in Tractatus Universi luris XI, I, Venezia, 1584

Tommaso Grammatico: Decisiones, Venezia, 1551

Tiberio Deciani: Tractatus criminalis, I, Torino, 1593

\section{Bibliografia}

Alessi G., 1979: Prova legale e pena. La crisi del sistema tra Evo medio e moderno, Napoli, Jovene

Baldwin J. W., 1994: The crisis of the Ordeal. Literature, Law and Religion about 1200, in "Journal of Medieval and Reinassance Studies", 24, pp. 327-353

Bartlett R., 1986: Trial by fire and water. The medieval judicial ordeal, Oxford, Clarendon Press

Bassani A., 2012: Sapere e credere. Parte prima. La veritas del testimone de auditu alieno dall'alto medioevo al diritto comune, Milano, Giuffrè

Bassani A., 2017: Udire e provare. I testimoni de auditu alieno nel processo di diritto comune, Milano, Giuffrè

Carbasse J. M., 2000: Histoire du droit pénal et de la justice criminelle, Paris, Presses Universitaires de France

Cavanna A., 1999: La "coscienza del giudice" nello stylus iudicandi del Senato di Milano, in Studi di storia del diritto, II, pp. 581-626 (ora in Cavanna A., 2007: Scritti 1968-2002, II, Napoli, Jovene, pp. 1037-1068)

Cerrito M., 2020: Pena negoziata e arbitrium iudicis. Le transazioni criminali nel Regnum Siciliae (secc. XIII-XV), Bologna, Bononia University Press

Chiodi G., 2013: Nel labirinto delle prove legali: la testimonianza del complice nel processo penale d'età moderna, in "Rivista internazionale di Diritto Comune", 24, pp. 113- 179

Chiodi G., 2014: Tortura 'in caput alterius', confessione 'contra alios' $e$ testimonianza del correo nel processo criminale medievale. Nascita e primi sviluppi dei criteri del diritto comune (secoli XII-XIV), in D. Mantovani, A. Padoa Schioppa (eds.), Interpretare il Digesto. Storia e metodi, Pavia, luss Press, pp. 673-728

Chiodi G., 2016: Crimini enormi e tortura ex processu informativo: una violazione dei diritto di difesa dell'imputato?, in "Glossae", 13, pp. 72-107

Chiodi G., 2017: Ad praesumptionem o ad plenam fidem? II valore probatorio 
della testimonianza del complice nel diritto canonico medievale, in "Italian Review of Legal History", 2, pp. 1-37

Chiodi G., 2018: La costituzione Qualiter et quando (c.8) e l'ordo inquisitionis nella canonistica medievale, in A. Larson, A. Massironi (eds.), The Fourth Lateran Council and the Development of Canon Law and the ius commune, Tournhout, Brepols, pp. 281-305

Condorelli O., 2013a: voce Antonio da Budrio, in Dizionario Biografico dei Giuristi Italiani, I, pp. 80-83

Condorelli O., 2013b: voce Niccolò Tedeschi, in Dizionario Biografico dei Giuristi Italiani, II, pp. 1426-1429

Condorelli O., Roumy F., Schmoeckel M. (eds.), 2009: Der Einfluss der Kanonistik auf die europäische Rechtskultur. Bd. I: Zivil- und Zivilprozessrecht, Köln, Bohlau

Condorelli O., Roumy F., Schmoeckel M. (eds.), 2012: Der Einfluss der Kanonistik auf die europäische Rechtskultur. Bd. III: Straf- und Strafprozessrecht, Köln, Bohlau

Condorelli O., Mausen Y., Roumy F., Schmoeckel M. (eds.), 2014: Der Einfluss der Kanonistik auf die europäische Rechtskultur. Bd. IV: Prozessrecht, Köln, Bohlau

Cortese E., 1978: Nicolaus de Ursone de Salerno. Un'opera ignota sulle lettere arbitrarie angioine nella tradizione dei trattati sulla tortura, in Per Francesco Calasso. Studi degli allievi, Roma, Bulzoni, pp. 191-284

Cortese E., 2008: Casi di giustizia sommaria: le lettere angioine, in P. A. Bonnet, L. Loschiavo (ed.), Forme stragiudiziali e straordinarie di risoluzione delle controversie nel diritto comune e nel diritto canonico. Atti del Convegno di studi, Teramo 21-22 aprile 2004, Napoli-Roma, Edizioni Scientifiche Italiane, pp. 7990

Cortese E., 2013: voce Paolo di (da) Castro, in Dizionario Biografico dei Giuristi Italiani, II, pp. 1505-1507

Cortese E., Pennington K., 2013: voce Pietro d'Ancarano, in Dizionario Biografico dei Giuristi Italiani, II, pp. 1578-1580

D’Amelio G., 1979: voce Castro, Paolo di, in Dizionario Biografico degli Italiani, 22

Del Re N., 1999: Prospero Farinacci giureconsulto romano (1544-1618), Roma, Fondazione Marco Besso

Di Renzo Villata M. G., 1996: Egidio Bossi. Un grande criminalista milanese quasi dimenticato, in Ius Mediolani. Studi di storia del diritto milanese offerti dagli allievi a Giulio Vismara, Milano, Giuffrè, pp. 365-615

Di Renzo Villata M. G., 2000: Felino Sandeicriminalista, in M. Bellomo, O. Condorelli (eds.), Proceedings of the Eleventh International Congress of Medieval Canon Law. Catania, 30 July - 6 august 2000, Città del Vaticano, Biblioteca Apostolica Vaticana, pp. 307-331 
Dezza E., 1989: Accusa e inquisizione. Dal diritto comune ai codici moderni, I, Milano, Giuffrè

Dezza E., 2013: Lezioni di storia del processo penale, Pavia, Pavia University Press

Durand B., 1993: Arbitraire du juge et consuetudo delinquendi: la doctrine pénale en Europe du XVI au XVIII siècle, Montpellier, Société d' histoire du droit et des institutions des anciens pays de droit écrit

Durand B., Otis-Cour L. (eds.), 2002: La torture judiciaire: approches historiques et juridiques, Lille, Centre d'histoire judiciaire

Elemér B., 2009: Die Verdachtsstrafe in Deutschland im 19. Jahrhunderts, Berlin, LIT

Engelmann W., 1922: Irrtum und Schuld nach der italienischen Lehre und Praxis des Mittelalters, Berlin, Scientia

Fiorelli P., 1954: La tortura giudiziaria nel diritto comune, Milano, Giuffrè

Fiori A., 2009: Praesumptio violenta o iuris et de iure? Qualche annotazione sul contributo canonistico alla teoria delle presunzioni, in Condorelli O., Roumy F., Schmoeckel M. (eds.), Der Einfluss der Kanonistik auf die europäische Rechtskultur. Bd. I: Zivil- und Zivilprozessrecht, Köln, Bohlau, pp. 75-106

Fiori A., 2012: 'Quasi denunciante fama': note sull'introduzione del processo tra rito accusatorio e inquisitorio, in O. Condorelli, F. Roumy, M. Schmoeckel (eds.), Der Einfluss der Kanonistik auf die europäische Rechtskultur. Bd. III: Straf- und Strafprozessrecht, Köln, Bohlau, pp. 351-367

Fiori A., 2013: Il giuramento di innocenza nel processo canonico medievale. Storia e disciplina della "purgatio canonica", Frankfurt a.M., Klostermann

Fiori A., 2016: Et si haereticus non sit... La condanna dei sola suspicione notabiles", in "Rivista internazionale di Diritto Comune", 27, pp. 185-225

Fiori A., 2020: La decretale Si culpa tua e la responsabilità degli enti morali nel diritto canonico classico", in Baura E., Puig Sanamuja F. (eds.), La responsabilità giuridica degli enti ecclesiastici, Milano, Giuffrè, pp. 33-76

Fraher R. M., 1989: Conviction According to Conscience: The Medieval Jurists' Debate concerning Judicial Discretion and the Law of Proof, in "Law and History Review", 7, pp. 23-88

Fraher R. M., 1992: IV Lateran's revolution in criminal procedure. the birth of "inquisitio", the end of ordeals, and Innocent III's vision of ecclesiastical politics, in R. J. Castillo Lara (ed.), Studia in honorem eminentissimi cardinalis Alphonsi M. Stickler, Roma, Libreria Ateneo Salesiano, pp. 97-111

Garlati L., 2004: Il diabolico intreccio. Reo convinto e indizi indubitati nel commento di Bartolomeo da Saliceto (C.4.19.25): alle radici di un problema, in Condorelli O. (ed.), Panta rei. Studi dedicati a Manlio Bellomo, II, Roma, II Cigno, pp. 387-419

Garlati L., 2011: II 'grande assurdo': la tortura del testimone nelle pratiche d'età moderna, in "Acta Histriae", 19, pp. 81-104 
Garlati L., 2016: Per una storia del processo penale: le pratiche criminali, in "Rivista di storia del diritto italiano", 89, pp. 71-109

Gouron A., 1990: Aux racines de la théorie des présomptions, in "Rivista internazionale di Diritto Comune", 1, pp. 99-109

Gouron A., 1992: Théorie des presomptions et pouvoir législatif chez les glossateurs, in Krynen J., Rigaudière A. (eds.), Droits savants et pratiques françaises du pouvoir (XI-XVe siècles), Bordeaux, Presses Universitaires de Bordeaux, pp. 117-127 (ora in Gouron A., 2000, III)

Gouron A., 1995a: Placentinus 'Herold' der Vermutungslehre?, in Festschrift zum 65. Geburtstag und zur Emeritierung von Professor Dr. Hans Kiefner, Münster, Inst. f. Kirchenrecht, pp. 90-103 (ora in Gouron A., 2000, VIII)

Gouron A., 1995b: Testis unus, testis nullus dans la doctrine juridique de XIle siècle, in Medievalia Lovanensia, ser. 1, 24, pp. 83-93 (ora in Gouron A., 2000, IX)

Gouron A., 2000: Juristes et droits savants: Bologne et la France médiévale, Aldershot, Ashgate

Kaser M., Hackl K., 1996: Das römische Zivilprozeßrecht, München, C.H. Beck

Kéry L., 2001: Inquisitio - denunciatio - exceptio: Möglichkeiten der Verfahrenseinleitung im Dekretalenrecht, in "Zeitschrift der Savigny-Stiftung für Rechtsgeschichte: Kanonistische Abteilung", 87, pp. 226-268

Landau P., 2012: 'Ne crimina maneant impunita'. Zur Entstehung des öffentlichen Strafanspruchs in der Rechtswissenschaft des 12. Jahrhunderts, in Der Einfluss der Kanonistik auf die europäische Rechtskultur. Bd. III: Straf- und Strafprozessrecht, Köln, Bohlau, pp. 25-35

Langbein J., 1977: Torture and the Law of Proof. Europe and England in the Ancien Regime, Chicago, University of Chicago Press

Lesne-Ferret M., 1996: Arbitraire des peines et droit coutumier dans le Midi de la France: le cas de Montpellier, in Studi di storia del diritto, I, Milano, pp. 27-47

Lévy J. Ph., 1939: La hiérarchie des preuves dans le droit savant du moyen-age depuis la Reinassance du Droit Romain jusqu'à la fin du XIVe siècle, Paris, Librairie du Recueil Sirey

Lévy J. Ph., 1965: L'évolution de la preuve dès origines à nos jours, in Recueils de la Société Jean Bodin pour I'histoire comparative des institutions, La preuve, II, Moyen Âge et Temps modernes, vol. XVII, Bruxelles, pp. 9-70

Mausen Y., 2006: Veritatis adiutor. La procèdure du tèmoinage dans le droit savant et la practique française (XIle - XIVe siècles), Milano, Giuffrè

Mazzacane A., 2013: voce Farinacci, Prospero, in Dizionario Biografico dei Giuristi Italiani, I, pp. 822-825

McAuley F., 2006: Canon Law and the End of the Ordeal, in "Oxford Journal of Legal Studies", 26, 3, pp. 473-513 
Meccarelli M., 1998: Arbitrium. Un aspetto sistematico degli ordinamenti giuridici in età di diritto comune, Milano, Giuffrè

Meccarelli M., 2002: Tortura e processo nei sistemi giuridici dei Territori della Chiesa. II punto di vista dottrinale (secolo XVI), in Durand B., Otis-Cour L. (eds.), La torture judiciaire: approches historiques et juridique, Lille, Centre d'histoire judiciaire, pp. 677-707

Melloni A., 2013: voce Sinibaldo Fieschi (Innocenzo IV, Papa), in Dizionario Biografico dei Giuristi Italiani, II, pp. 1872-1874

Miletti M. N., 2015: Le pratiche criminali di Pietro Follerio. Giustizia e poteri nel Mezzogiorno italiano del Cinquecento, in M. Torres Aguilar, M. Pino Abad (eds.), Burocracia, poder político y justicia. Libro-homenaje de amigos del profesor José María García Marín, Madrid, Dykinson, pp. 495-530

Motzenbäcker R., 1958: Die Rechtsvermutung im kanonischen Recht, München, K. Zink

Padoa Schioppa A., 1967: Unus testis, nullus testis: note sulla scomparsa di una regola processuale, in Studia Ghisleriana, pp. 334-357 (ora in Padoa Schioppa A., 2003: Italia ed Europa nella storia del diritto, Bologna, II Mulino, pp. 460484)

Padovani A., 2013: voce Tommaso da Piperata (di Peverata, degli Sturlitti), in Dizionario Biografico dei Giuristi Italiani, II, pp. 1959-1960

Padovani A., 2017: Dall'alba al crepusculo del commento: Giovanni da Imola (1375 ca. - 1436) e la giurisprudenza del suo tempo, Frankfurt a.M., Klostermann

Paravicini Bagliani A., 2004: voce Innocenzo IV, papa, in Dizionario Biografico degli Italiani, vol. 62, pp. 438 ss.

Pennington K., 1993: voce Enrico da Susa, detto l'Ostiense, in Dizionario Biografico degli italiani, 42, pp. 758 ss.

Provera G., 1979: Prova, sentenza, appello in diritto romano, in Atti del colloquio romanistico-canonistico (febbraio 1978), Roma, Pontificia Università Lateranense, pp. 391-399 (ora in Provera G., Scritti giuridici https://www. ledonline.it/rivistadirittoromano/allegati/proveraprova.pdf)

Quaglioni D., 2013: voce Gandino, Alberto, in Dizionario Biografico dei Giuristi Italiani, I, pp. 942-944

Rosoni I., 1995: Quae singula non prosunt collecta iuvant. La teoria della prova indiziaria nell'età medievale e moderna, Milano, Giuffrè

Roth A., 1998: voce Verdachtsstrafe, in Handwörterbuch zur deutschen Rechtsgeschichte, 5, pp. 681-684.

Russo M., 2017: Prova legale e libero convincimento del giudice, Torino, Giappichelli

Salvioli G., 1927: Storia della procedura civile e criminale, in Del Giudice P. (ed.), 
Storia del diritto italiano, III, 2, Milano, Hoepli

Sbriccoli M., 1998: "Vidi communiter observari". L'emersione di un ordine penale pubblico nelle città italiane del secolo XIII, in "Quaderni fiorentini per la storia del pensiero giuridico moderno", 27, pp. 231-268

Sbriccoli M., 1991: Tormentum, id est torquere mentem. Processo inquisitorio e interrogatorio per tortura nell'Italia comunale, in Maire Vigueur J. C., Paravicini Bagliani A. (eds.), La parola dell'accusato, Palermo, Sellerio, pp. 17-32 (ora in Sbriccoli M., 2009, Storia del diritto penale e della giustizia. Scritti editi e inediti (1972-2007), I, Milano, Giuffrè, pp. 111-128)

Schaffstein F., 1989: Verdachtsstrafe, außerordentliche Strafe und Sicherungsmittel im Inquisitionsprozeß des 17. und 18. Jahrhunderts, in Zeitschrift für die gesamte Strafrechtswissenschaft, 101, pp. 493-515

Schmoeckel M., 2000: Humanität und Staatsraison. Die Abschaffung der Folter in Europa und die Entwicklung des gemeinen Strafprozess- und Beweisrechts seit dem Hohen Mittelalter, Köln, Bohlau

Schmoeckel M., 2002: La survivance de la torture après la chute de l'Empire romain jusqu'à l'aube du lus Commune, in Durand B., Otis-Cour L. (eds.), La torture judiciaire: approches historiques et juridique, Lille, Centre d'histoire judiciaire, pp. 315-329

Schnapper B., 1973: Peines arbitraires du XIIle au XVIIIe siècle (doctrines savantes et usages francais), in Tijdschrift voor rechtsgeschiedenis, 41, pp. 237-277

Thäle B., 1993: Die Verdachtsstrafe in der kriminalwissenschaftlichen Literatur des 18. und 19. Jahrhunderts, Frankfurt a. M., Peter Lang

Treggiari F., 2007: La fides dell'unico teste, in Prodi P. (ed.), La fiducia secondo $i$ linguaggi del potere, Bologna, II Mulino, pp. 53-72

Trusen W., 1988: Der Inquisitionsprozeß. Seine historischen Grundlagen und frühen Formen, in "Zeitschrift der Savigny-Stiftung für Rechtsgeschichte: Kanonistische Abteilung", 74, pp. 168-230

Vallerani M., 2005: La giustizia pubblica medievale, Bologna, II Mulino

Vallerani M., 2009: I/ giudice e le sue fonti. Note su inquisitio e fama nel Tractatus de maleficiis di Alberto da Gandino, in "Rechtsgeschichte. Zeitschrift des MaxPlanck-Instituts für europäische Rechtsgeschichte", 14, pp. 40-61

Vallerani M., 2010: L'arbitrio negli statuti cittadini del Trecento. Note comparative, in M. Vallerani (ed.), Tecniche di potere nel tardo medioevo. Regimi comunali e signorie in Italia, Roma, Viella, pp. 117-148

Varalda C. E., 2016: II contributo di Innocenzo III alla formazione della cultura giuridica occidentale: in particolare in relazione al noto principio 'rei publicae interest ne crimina remaneant impunita', in "Vergentis: revista de investigación de la Cátedra Internacional conjunta Inocencio III", 3, pp. 144-169 